Fumiya Kitayama and Ryou Kondo

\title{
Development of a two degrees-of-freedom linear oscillatory actuator for vibration control
}

ABSTRACT. In automobiles, several mechanical parts generate undesirable multidimensional vibrations, which are usually actively controlled by using one degree-of-freedom (DOF) linear oscillatory actuators. This paper proposes a 2-DOF linear oscillatory actuator to realize active control with high vibration control performance and downsizing. First, the design specifications, basic construction, and operation principle were established. Finite element analysis and MATLAB/Simulink were used to confirm that the resulting characteristics satisfied the specification requirements. Finally, the characteristics determined using a prototype were compared with those determined in the analysis.

Keywords: dynamic characteristics, inertial force, multiple degrees-of-freedom

Nanotechnology Perceptions 15 (2019) 212-219

doi: 10.4024/N23KI19A.ntp.15.03 